\title{
A RAPID CYCLING HYDROGEN BUBBLE CHAMBER WITH HIGH SPATIAL RESOLUTION FOR VISUALIZING CHARMED PARTICLE DECAYS
}

\author{
J.L. BENICHOU, A. HERVÉ, H. LEUTZ, G. PASSARDI, W. SEIDL, J. TISCHHAUSER, H. WENNINGER \\ CERN, Geneva, Switzerland
}

and

C.M. FISHER

Rutherford Laboratory, Chilton, England

Received 23 June 1981

For the purpose of directly detecting the decays of charmed particles, a rapid cycling hydrogen chamber with high spatial resolution has been constructed. In several runs it was exposed to $360 \mathrm{GeV}$ proton and negative pion beams and has taken a total of 1.63 million pictures during more than 20 million expansion cycles. The resolved bubble diameters were around $40 \mu \mathrm{m}$ with 80.5 counted bubbles $/ \mathrm{cm}$ at $33 \mathrm{~s}^{-1}$ chamber and $17 \mathrm{~s}^{-1}$ camera cycling rates.

\section{Introduction}

Charm is a quantum number $(C)$ which is assigned to a new class of particles of comparatively high restmass $\left(>1.86 \mathrm{GeV} / c^{2}\right)$. In strong and electromagnetic interactions $C$ is conserved and therefore charmed particles are produced as associated pairs by hadrons and photons. In weak interactions $C$ is not conserved, therefore charmed particles are produced singly by neutrinos, and decay weakly like strange particles. The most probable decay topologies for the ground state mesons are 2 and 4 prong $\mathrm{V}^{0}$ 's for the neutral states $\left(D^{0} ; \bar{D}^{0}\right)$ and 1 and 3 prong decays for the charged states $\left(\mathrm{D}^{+} ; \mathrm{D}^{-}\right)$.

Mass values and decay modes for the ground states of charmed particles have been established from production with $\mathrm{e}^{+}, \mathrm{e}^{-}$colliding beams at SLAC [1] and at DESY [2]. Decay lengths observed with emulsion exposures in neutrino [3] and in photon beams [4] indicate lifetimes in the range $10^{-12}-10^{-13} \mathrm{~s}$. Finally, the excess of neutrino events in beam dump experiments [5] provides evidence for hadronic charm production with cross sections ranging from $10-50 \mu \mathrm{b}$ at around $400 \mathrm{GeV}$ proton energy.

In contrast to production via the weak interaction, hadronic interactions produce associated charmed pairs. Such events are topologically complex, because of the short distances involved in the two charmed decays and the associated high multiplicity of other particles which frequently include strange mesons and hyperons decaying also close to the production vertex. The direct visual observation of charmed decays is the only clean method of separating these events from background and hence allowing a detailed study of their production and decay properties.

A rapid cycling bubble chamber with high spatial resolution could be well suited as a charm sensitive vertex detector for hadron and photon experiments. Filled with hydrogen, such a bubble chamber would allow one to study the relatively simple production process involving only a single free proton, and the absence of neutrons would provide unambiguous discrimination between charged charm decays (odd prongs) and secondary interactions (even prongs). Combined with a particle spectrometer, to analyze the tracks emerging from the photographed interaction vertices, this visual vertex detector would represent a unique tool for the study of charmed particle production, decay and spectroscopy. This paper reports on the design, construction and operation of such a bubble chamber, named LEBC, an acronym for LExan Bubble Chamber.

LEBC has already completed two physics experiments. In the first exposure (NA13) it was operated, without any auxiliary detector, to search for topolog- 
ical evidence for charmed particles produced in 340 $\mathrm{GeV}$ negative pion interactions [6]. During the second experiment (NA16), as the track sensitive vertex detector of the European Hybrid Spectrometer (EHS), it was exposed to a negative pion and to a proton beam. The spectrometer provided momentum analysis, particle identification and gamma detection for the reconstruction of forward emitted secondaries, in particular decay products identified as charm candidates on LEBC pictures.

\section{Design considerations}

The basic parameters for a detector intended to visualize charm decays are established by the lifetimes and cross sections of such particles: the lifetimes dictate the spatial resolution required to detect their typical decay patterns within the complex beam interaction region. Their production cross sections determine the sensitivity of the detector, necessary to collect a meaningful sample of charmed candidates within a reasonable exposure time.

The problem of selecting charm decays via topological information has been discussed already in ref. 7 and is illustrated by fig. 1: a particle of rest mass $m_{0}$, produced at an angle $\theta_{p}$ with momentum $p$, transverse momentum $p_{\mathrm{t}}$, and decaying after a lifetime $\tau$ yields the decay length

$L=p \tau / m_{0}=p_{t} \tau /\left(m_{0} \sin \theta_{p}\right)$.

Assuming $p_{\mathrm{t}}<m_{0} c=1.86 \mathrm{GeV} / c$ (for charged and neutral $\mathrm{D}$ mesons) and $\tau \leqslant 10^{-12} \mathrm{~s}$ the transverse distance $L \sin \theta_{p}$ of the decay vertex from the beam direction will always be less than $300 \mu \mathrm{m}$. For the shorter lived neutral D mesons this distance will be

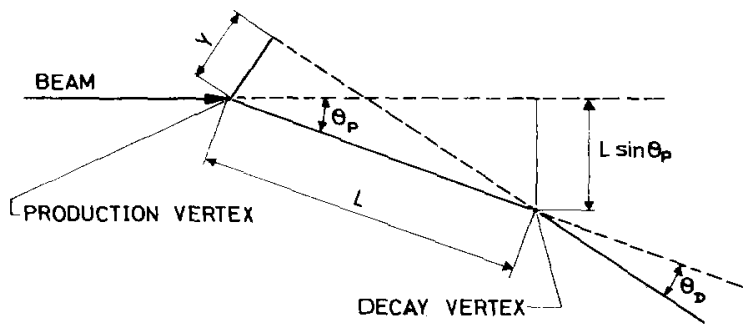

Fig. 1. Topological information from a $D$ meson produced at an angle $\theta_{p}$ and decaying after a distance $L$ at an angle $\theta_{\mathrm{D}}$ The transverse separation $L \sin \theta_{p}<c \tau(\tau=$ lifetime) of the decay vertex from the beam direction is independent of the longitudinal momentum. The impact parameter $y=L \sin \theta_{\mathrm{D}}$ is defined by decay kinematics and approaches $c \tau$. even less than $30 \mu \mathrm{m}$. Note, that this is independent of the longitudinal momentum $p$ and hence of the decay length $L$ which, for production at high forward projection, in a $400 \mathrm{GeV}$ interaction can be several centimetres for charged $\mathrm{D}$ mesons and several millimetres for $\mathrm{D}^{0}, \Lambda_{\mathrm{c}}^{+}$etc. The charm decay will therefore in general be buried in the forward cone of secondaries. It is only detected by the observation of a track which has a significant impact parameter and therefore misses the production vertex. This impact parameter $y$ in fig. 1 is defined by the kinematics of the decay (index D)

$y=L \sin \theta_{\mathrm{D}}=p \tau\left(p_{\mathrm{t}} / p\right)_{\mathrm{D}} / m_{0} \sim c \tau$.

From the Monte-Carlo study reported in ref. 7, we find that to detect $\mathrm{D}^{+}$decays with reasonable efficiency it is necessary to resolve bubble diameters smaller than $50 \mu \mathrm{m}$. At the same time correspondingly high bubble densities are necessary, to clearly identify particle tracks composed of such small bubbles.

To resolve these small bubbles, we need an optical system with a resolution $R$ better than $50 \mu \mathrm{m}$. Unfortunately, the depth of field $d$, which defines the liquid layer where the tracks are well in focus, is linked with the resolution via the Rayleigh criterion

$R=0.61 \sqrt{ } d \lambda$.

At a wavelength $\lambda$ of $550 \mathrm{~nm}$ we expect only 12.2 , $7.8,4.4$ and $2 \mathrm{~mm}$ depth of field for resolutions of $50,40,30$ and $20 \mu \mathrm{m}$, respectively. Since the depth of field is not sharply defined, particle tracks may still be recognized beyond the Rayleigh limit, but the incoming particle beam and hence the production and decay vertices of $\mathrm{D}$ mesons must be contained in this zone.

Assuming $40 \mathrm{~mm}$ depth of chamber liquid, we cover more than three times the depth of field associated with $50 \mu \mathrm{m}$ resolution. This comparatively small chamber depth would have the advantage, that inevitable slight imhomogeneities in the liquid temperature barely deviate the light rays in their short passage through the chamber. In addition, it requires only a small piston stroke for the expansions, but increases considerably the surface to volume ratio of the chamber and, therefore, the radiative heat load per unit of liquid volume.

The expected nominal cross section for hadronic charm production must be multiplied by the detection efficiency for charm decays to obtain the observable cross section. This detection efficiency depends 
on the lifetime of charmed particles and on the spatial resolution of the detector, in our case on the resolved bubble size. The Monte-Carlo study [7] estimates an observable cross section of $5 \mu \mathrm{b}$, assuming an inclusive D production cross section of $40 \mu \mathrm{b}$ and lifetimes of $10^{-12} \mathrm{~s}$ and $10^{-13} \mathrm{~s}$ for $\mathrm{D}^{ \pm}$and $\mathrm{D}^{0}$, respectively. Therefore we expected to find one charmed pair within 4000 beam interactions. This poor signal raises immediately the question of background processes simulating charm decays and hence the nature of the detector liquid becomes important. In hydrogen, the absence of neutrons allows unambiguous discrimination between charged charm decays (odd prongs) and interactions (even prongs). Moreover, we expect high production multiplicities and hence, in a denser target medium, considerable interaction and gamma conversion probabilities. Again, the comparatively large interaction and conversion length in hydrogen keep the vertex region relatively free from such secondary events. In addition, in hydrogen there is the obvious attraction to study the simple production process involving only a single free proton. Because of these clear advantages, we chose hydrogen as the chamber liquid, in spite of the complications associated with cryogenics and hydrogen safety.

If we aim at a sample of about 100 observed charmed pairs within a 10 day exposure, we require five photographed beam interactions per second. This rate $i$ is based on $15 \%$ accelerator duty cycle and $60 \%$ data taking efficiency. It depends on the beam interaction probability $p$ in hydrogen, the expansion frequency $f$ of the chamber and the maximum camera rate $f / k$ :

$i=p f /[1+p(k-1)]$.

With a chamber dimension $s=20 \mathrm{~cm}$ along the beam and $n=15$ tracks per picture, we obtain $p=1$ $\exp [-(n s / l)] \sim 0.2$ for an interaction length, $l=14 \mathrm{~m}$, in hydrogen at $29 \mathrm{~K}$. To efficiently correlate the dead times between chamber expansions and film exposures, the expansion ratio $k$ must be an integral number. The photographed event rates $i$ from relation (3) are listed as a function of the expansion frequency $f$ and the ratio $k$ of expansion and camera rate in table 1 . To avoid picture taking with no events on, an interaction trigger is required whose logic indicates the events and starts the timing for bubble growth. Since the trigger counters must be placed up and downstream the chamber vacuum tank, they trigger also on events in the relevant beam windows. The
Table 1

Rate of photographed events at 0.2 interaction probability in liquid hydrogen in dependence from chamber frequency $f$ and the integer number $k$ of the camera rate $f / k$ (eq. (3)].

\begin{tabular}{llllll}
\hline$f$ & $k:$ & & & & \\
\cline { 2 - 6 } & 1 & 2 & 3 & 4 & 5 \\
\hline 20 & 4.0 & 3.3 & 2.9 & 2.5 & 2.2 \\
25 & 5.0 & 4.2 & 3.6 & 3.1 & 2.8 \\
30 & 6.0 & 5.0 & 4.3 & 3.8 & 3.3 \\
35 & 7.0 & 5.8 & 5.0 & 4.4 & 3.9 \\
40 & 8.0 & 6.7 & 5.7 & 5.0 & 4.4 \\
45 & 9.0 & 7.5 & 6.4 & 5.6 & 5.0 \\
50 & 10.0 & 8.3 & 7.1 & 6.3 & 5.6 \\
\hline
\end{tabular}

window thickness, in terms of interaction length, must be compared with the $20 \mathrm{~cm}$ of liquid hydrogen and the event rate attributed to them should stay below $50 \%$ of the total trigger rate.

The bubble size to be photographed can be selected by appropriate choice of its growth time, i.e. the time delay between the interaction trigger signal and the firing of the flash light. To avoid flash delays shorter than $100 \mu \mathrm{s}$, which would approach the flash duration time, it is advisable to operate the chamber at much higher hydrogen temperatures than those usually adopted. As can be seen from the pressuretemperature diagram in fig. 2 , the minimum pressure at $29 \mathrm{~K}$ is much higher than at $26 \mathrm{~K}$. This increases the amount of vapour required for the same bubble size and hence slows down the growth rate considerably.

High bubble densities can be achieved by expanding the liquid as closely as possible to its foam limit, which is indicated in fig. 2 . The foam limit separates the $p-T$ area, where finite bubble formation energies are required, from that $p-T$ area, where free bubble formation due to the vanishing liquid surface tension occurs in the bulk liquid. In conventional chambers the approach to this foam limit is normally prevented by parasitic boiling at spots, where some surface defects favour cavitation, in particular at the window and piston seals. Subsequent $p V$-work on these parasitic bubbles will cause them to grow and hence the compressibilitiy of the chamber filling will increase. This will increase the minimum pressure until a stable condition well above the foam limit is re-established. To avoid this problem completely, we decided to 


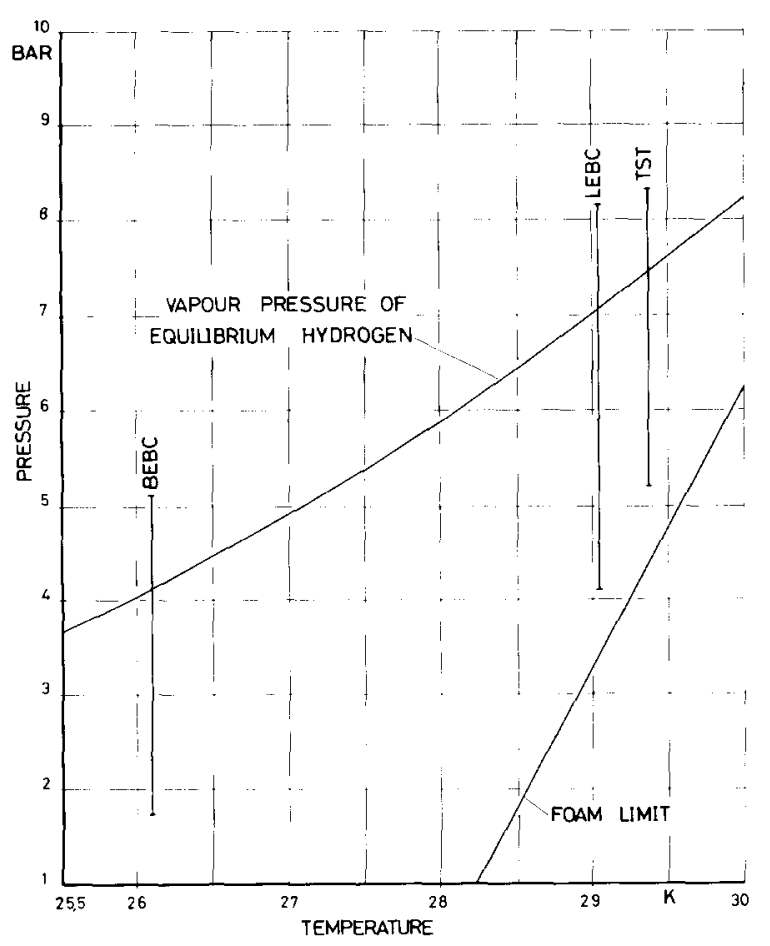

Fig. 2. Pressure-temperature diagram for liquid equilibrium hydrogen. The vertical bars indicate the pressure cycle between static and minimum pressure of the chamber liquid. The $40 \mathrm{~m}^{3}$ bubble chamber BEBC is an example for the normally adopted operating conditions. LEBC and TST (Track Sensitive Target immersed in $\mathrm{Ne}-\mathrm{H}_{2}$ ) indicate, that operation at rather high temperatures to produce small bubbles of high bubble density is feasible. The foam limit separates the two $p-T$ areas, where chamber operation is possible (above) and where free evaporation starts in the bulk liquid (below).

build the chamber body entirely from plastic material. Track sensitivity is achieved via a fixed circular membrane ratner than by a sliding piston. A plastic optics window is glued onto the chamber body and in this way a closed chamber volume without seals is obtained.

To summarize: For the envisaged search for charmed pairs a visual detector with at least $50 \mu \mathrm{m}$ spatial resolution and a photographed interaction rate of about 5 events $\mathrm{s}^{-1}$ is necessary, implying a hydrogen bubble chamber with high resolution optics, around $30 \mathrm{~s}^{-1}$ expansion and $15 \mathrm{~s}^{-1}$ camera rates. To achieve high bubble densities, the hydrogen has to be expanded, within a cycle time as short as feasible, close to its foam limit.

\section{The bubble chamber system}

\subsection{Chamber body}

To meet the above design criteria, we constructed a cylindrical chamber body with $20 \mathrm{~cm}$ inner diameter and $4 \mathrm{~cm}$ free depth (fig. 3 ). The chamber is made track sensitive by expanding the $1.1 \mathrm{\ell}$ volume via a $5 \mathrm{~mm}$ thick membrane, forming the rear flat end of the horizontal cylinder and supported across its outer surface by a hydraulically driven piston. Scotchlite is glued onto the inner surface of this membrane and particle tracks are photographed in bright field illumination through a $25 \mathrm{~mm}$ thick window, which is glued to the chamber cylinder and forms its front end plate.

In order to realize a "clean" chamber, i.e. with smooth surfaces and no joints, and to avoid different thermal contractions during cool down, all parts of the body had to be constructed from the same plastic material. For photography it must be transparent for visible light and to perform the several millions of short expansion cycles, the membrane material must tolerate sufficient elongation and posses high impact strength to resist the severe shocks caused by the high piston accelerations. From all the plastic materials available, only the thermoplastic polycarbonate Lexan offers these properties as is illustrated in table 2, which compares Lexan with Plexiglas the only other sufficiently transparent plastic.

To reduce the amount of glueing, the chamber cylinder with its $2 \mathrm{~mm}$ thick beam windows and the expansion membrane were machined from one single block of Lexan. This basic structure was then vacuum sealed to the Lexan optics window by solvent cementing the two parts along a $30 \mathrm{~mm}$ wide annular margin. We applied no adhesive bondings, and used no ultrasonic or heat seals, since these procedures result in rather weak and brittle mechanical joints, which fail at cryogenic temperatures. With solvent cementing we achieved the required vacuum tight joints of high structural integrity with smooth edges. The cementing technique used methylene chloride as a solvent at 70 bar pressure. Due to its low boiling point of $40^{\circ} \mathrm{C}$, this solvent completely escapes within a few days from the glued joint, which has the same mechanical properties as pure Lexan. Careful precautions were required to avoid splashing the solvent onto the membrane surface, thus causing microfissures, leading to fractural destruction after a few expansions.

To avoid light absorption in the optics window, we 


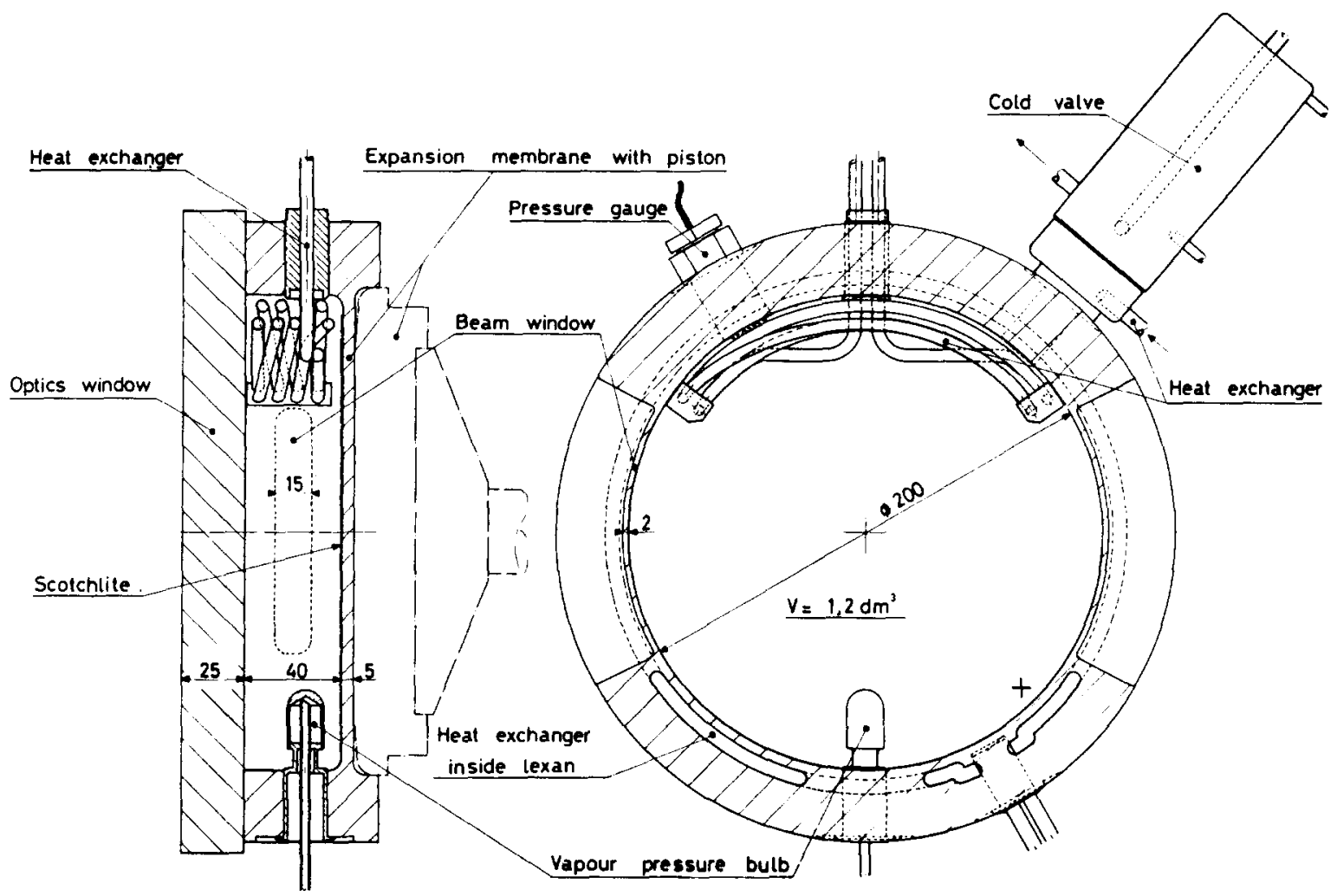

Fig. 3. Cross section of the LEBC chamber body.

Table 2

Some relevant data for Lexan and Plexiglas.

\begin{tabular}{|c|c|c|c|c|}
\hline Parameters & & Units & Lexan & Plexiglas \\
\hline Chemical denotation & & - & thermoplastic polycarbonate & polymethyl-methacrylate \\
\hline Chemical composition & & - & $-\left(\mathrm{H}_{14} \mathrm{C}_{16} \mathrm{O}_{3}\right)_{x^{-}}$ & $\left(\mathrm{H}_{8} \mathrm{C}_{4} \mathrm{O}_{2}\right)$ \\
\hline Density & $293 \mathrm{~K}$ & $\mathrm{gcm}^{-3}$ & 1.20 & 1.18 \\
\hline Radiation length & $293 \mathrm{~K}$ & $\mathrm{~cm}$ & 34.5 & 34.1 \\
\hline Interaction length & $293 \mathrm{~K}$ & $\mathrm{~cm}$ & 48.3 & 48.9 \\
\hline Refractive index (546 nm) & $\begin{array}{r}293 \mathrm{~K} \\
30 \mathrm{~K}\end{array}$ & & $\begin{array}{l}1.5902[8] \\
1.5682 \mathrm{c})\end{array}$ & $1.4970[8]$ \\
\hline $\begin{array}{l}\text { Thermal contraction } \\
\qquad 293 \mathrm{~K} \rightarrow\end{array}$ & $30 \mathrm{~K}$ & $\%$ & $1.2 \mathrm{~b})$ & $1.0 \mathrm{~b})$ \\
\hline $\begin{array}{l}\text { Modulus of } \\
\text { elasticity }\end{array}$ & $\begin{array}{r}293 \mathrm{~K} \\
77 \mathrm{~K}\end{array}$ & $\mathrm{kgcm}^{-2}$ & $\begin{array}{l}22000^{a)} \\
50500^{b)}\end{array}$ & $\begin{array}{l}33000 \text { a) } \\
85000^{b)}\end{array}$ \\
\hline $\begin{array}{l}\text { Elongation (yield) } \\
\text { Elongation (ultimate) }\end{array}$ & $\begin{array}{r}293 \mathrm{~K} \\
77 \mathrm{~K}\end{array}$ & $\begin{array}{l}\% \\
\%\end{array}$ & $\begin{array}{l}80 \text { a) } \\
\sim 20 \text { b) }\end{array}$ & $\begin{array}{l}5.5 \text { a) } \\
\sim 2 \text { b) }\end{array}$ \\
\hline $\begin{array}{l}\text { Impact strength } \\
\text { Imp. str. (notched) }\end{array}$ & $\begin{array}{l}293 \mathrm{~K} \\
293 \mathrm{~K}\end{array}$ & $\mathrm{cmkgcm}^{-2}$ & $\begin{array}{l}\text { Samples not broken a) } \\
>30 \text { a) }\end{array}$ & $\begin{array}{l}20 \text { a) } \\
2 \text { a) }\end{array}$ \\
\hline Tensile strength (yield) & $\begin{array}{r}293 \mathrm{~K} \\
77 \mathrm{~K}\end{array}$ & $\mathrm{kgcm}^{-2}$ & $\begin{array}{l}600 \text { a) } \\
\sim 1300 \text { b) }\end{array}$ & $\begin{array}{l}800 \text { a) } \\
\sim 1300 \text { a) }\end{array}$ \\
\hline Flexural strength (yield) & $\begin{array}{r}293 \mathrm{~K} \\
77 \mathrm{~K}\end{array}$ & $\mathrm{kgcm}^{-2}$ & $\begin{array}{l}900^{\text {a) }} \\
\sim 2000 \text { b) }\end{array}$ & $\begin{array}{l}1400^{a)} \\
\sim 2200^{b)}\end{array}$ \\
\hline
\end{tabular}

a) Data from Röhm, D-6100 Darmstad, Germany .

b) Data from CERN.

c) Extrapolated from ref. 8 . 
did not use normal Lexan with its blueish tint, but ordered a special type * without any tint and produced under stringent precautions against dirt contamination. Then, at another firm $* *$, four $1 / 4$ inch thick sheets of this raw material were sandwiched between two highly polished chromium plates and by a heating procedure at high pressure, patented as "press-polishing", amalgamated to a 1 inch thick plate. The result was an optical grade Lexan plate of high light transmittance, which did not show any of the traces from the extrusion process typical of Lexan (with $25 \mathrm{~mm}$ thickness we measured at 550 nm wavelength: $90 \%$ transmittance, $9 \%$ surface reflections, $1 \%$ absorption). Once the required Lexan material is available and the procedures for glueing and machining are well established, a spare chamber body can be manufactured within two weaks.

All metallic parts, such as the heat exchanger, pressure gauge, vapour pressure bulb, and filling valve (fig. 3) were mounted on the basic structure of the body before the glueing process. These metal-Lexan connections were vacuum sealed without any glueing by a shrinking technique: the stainless steel parts were cooled to liquid nitrogen temperature and fitted into appropriately dimensioned borings in the Lexan structure, which was warmed up to $45^{\circ} \mathrm{C}$.

The chamber body and all cold components of the cryogenic equipment were installed inside one common vacuum tank, as shown in fig. 4 . They were protected against radiant heat by hydrogen-gas cooled screens and appropriate layers of "super insulation". A turbomolecular pump provided a hydrocarbon free vacuum in the $10^{-6}$ mbar range.

\subsection{Expansion system}

The adiabatic pressure cycles which render the liquid hydrogen superheated by reducing its pressure and which recompress the bubbles formed, are generated by a hydraulically driven piston. Its action is transmitted to the chamber liquid via the expansion membrane. This membrane is fixed on its outer circular edge of radius $r_{0}$ and rests on the piston with a concentric area of radius $r$ (see insertion in fig. 5). This is in fact the active surface of the membrane and its area depends on the piston position and on the chamber pressure. To minimize the piston strokes and hence to keep the elongation of the Lexan-membrane

\footnotetext{
* SL3000 from General Electric Comp. Mount Vernon, Indiana, USA.

** Westlake Plastics Comp. Lenni, Pensylvania, USA.
}

as small as possible, the active area with the varying radius $r$ should be as large as possible. This can be achieved by carefully adjusting the mass of liquid hydrogen in the chamber in such a way that, at the operating temperature, the piston rest-position renders the membrane flat at $r=r_{0}$. During the first half of each expansion cycle the membrane moves away from its flat position and at the same time the chamber pressure decreases. Both variations decrease the active area of the membrane, as can be seen from fig. 5. In order to reach the bottom of the expansion cycle with a reasonable active area, the minimum pressure must be as high as possible. To achieve this condition, we operated LEBC at a comparatively high hydrogen temperature, as illustrated by the $p-T$ diagram in fig. 2. Fortunately, this condition coincides with that required to produce tracks with high bubble density at a reduced bubble growth rate, in order to keep the flash delay also for small bubble diameters reasonably long.

Once its filling value is closed, LEBC represents a thermodynamic system, where the static hydrogen pressure is determined by the rest position of the piston between the pressure cycles. Therefore, the expansion system acted as a positional electrohydraulic servoactuator, which compensated all static pressure variations, mainly due to hydrogen temperature flucturations, by automatic adjustments of the piston rest-position. A block diagram of the system is shown in fig. 6 . During the expansion cycles, the basic servo-loop A compares at point $\mathrm{C}_{\mathrm{A}}$ the actuator position $X_{\mathrm{p}}$ with an input signal from a function generator, which defines the desired shape of the cycle. Between the expansions, a second loop B compares at point $\mathrm{C}_{\mathrm{B}}$ the chamber static pressure $P_{\mathrm{s}}$, obtained from a full bridge strain gauge pressure transducer *, with a pre-set value. The action of loop B is suppressed by a "track and hold" expansion feature during the expansion cycles. The control force is provided by a double acting piston (fig. 7) of $11 \mathrm{~cm}^{2}$ area, driven by two parallel servo valves **. The end of the rod $\left(16 \mathrm{~cm}^{2}\right.$ area) serves as an auxiliary piston (fig. 7), which balances the force acting on the expansion membrane due to the hydrogen pressure. The main parameters of the expansion system are listed in table 3.

During all LEBC exposures this expansion motor performed outstandingly well and never gave rise to a

\footnotetext{
* Bell and Howell, Pasadena, California 91109, USA.

** Moog Inc. Proner Airport, East Aurora, New York 14052, USA.
} 
stop in data taking. It did not generate high frequency vibrations and we benefitted in particular from its flexibility when adjusting the cycle length and rate to achieve optimum operating conditions for LEBC.

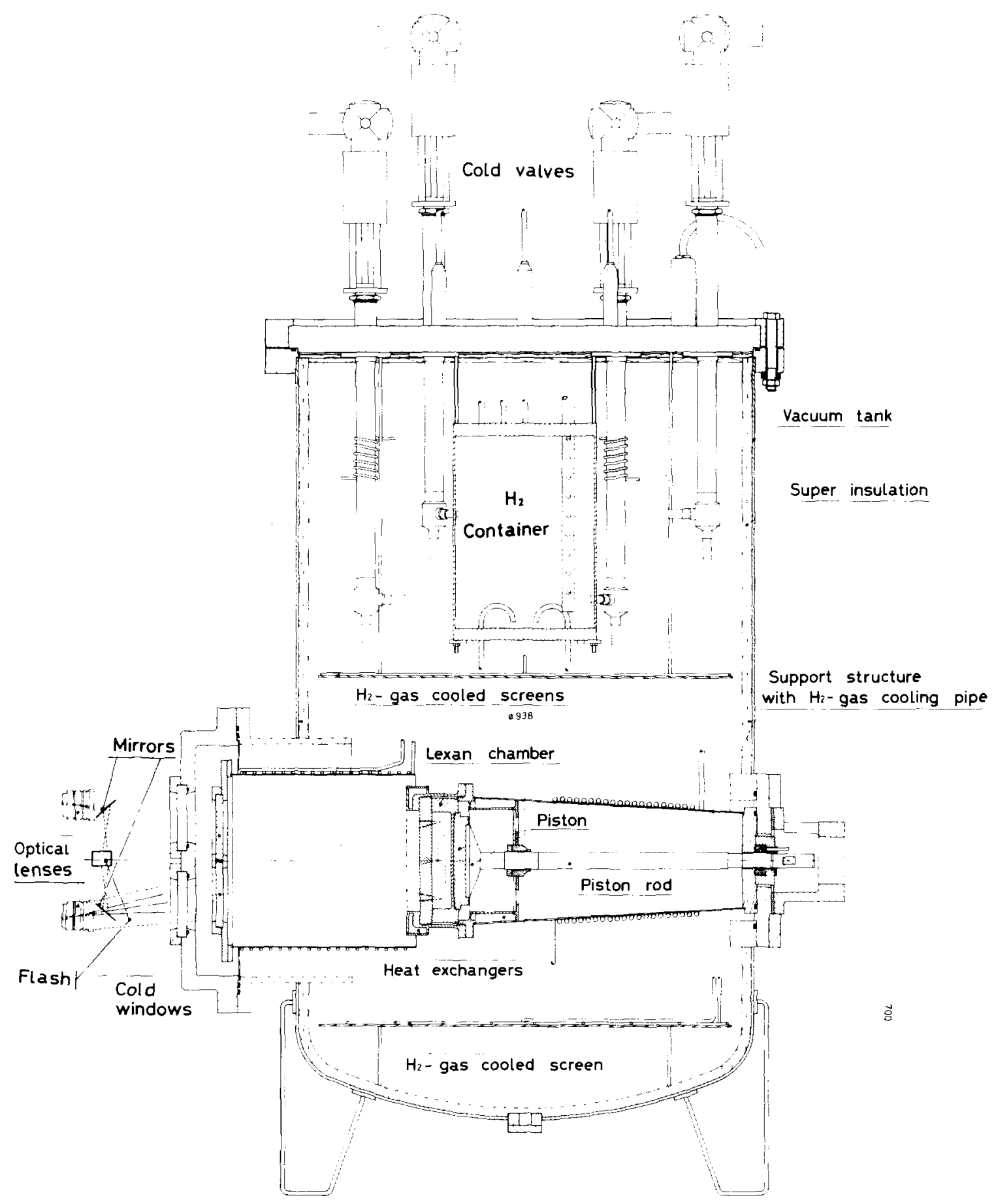

Fig. 4. Vertical section of the LEBC vacuum tank with the cryogenic equipment at the upper part and with chamber body, optics and expansion-system at the lower part. 


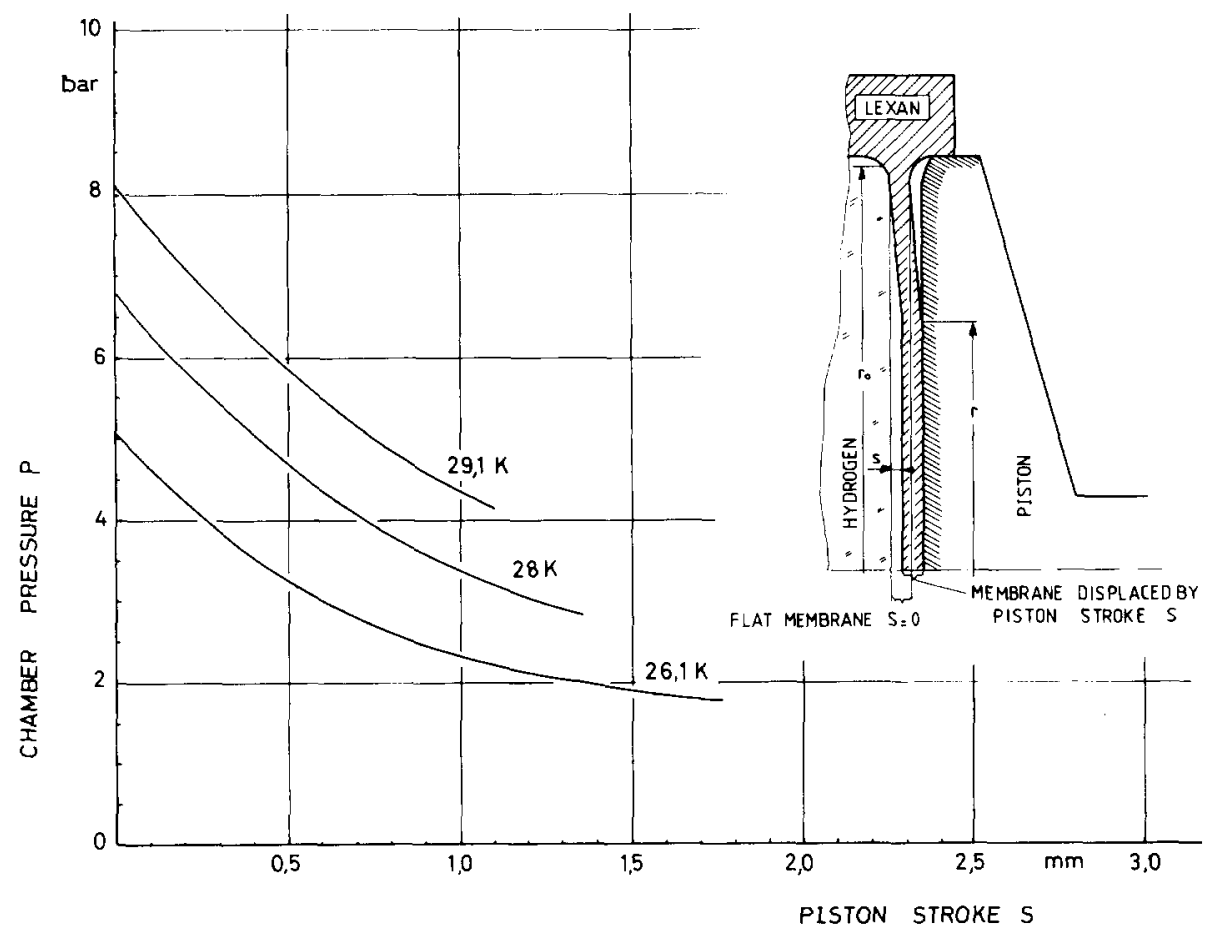

Fig. 5. Piston stroke required for the pressure cycles at the indicated hydrogen temperatures. The action of the piston is trans. mitted to the liquid hydrogen via an expansion membrane which is fixed, according to the shown insert, on its outer circular edge.

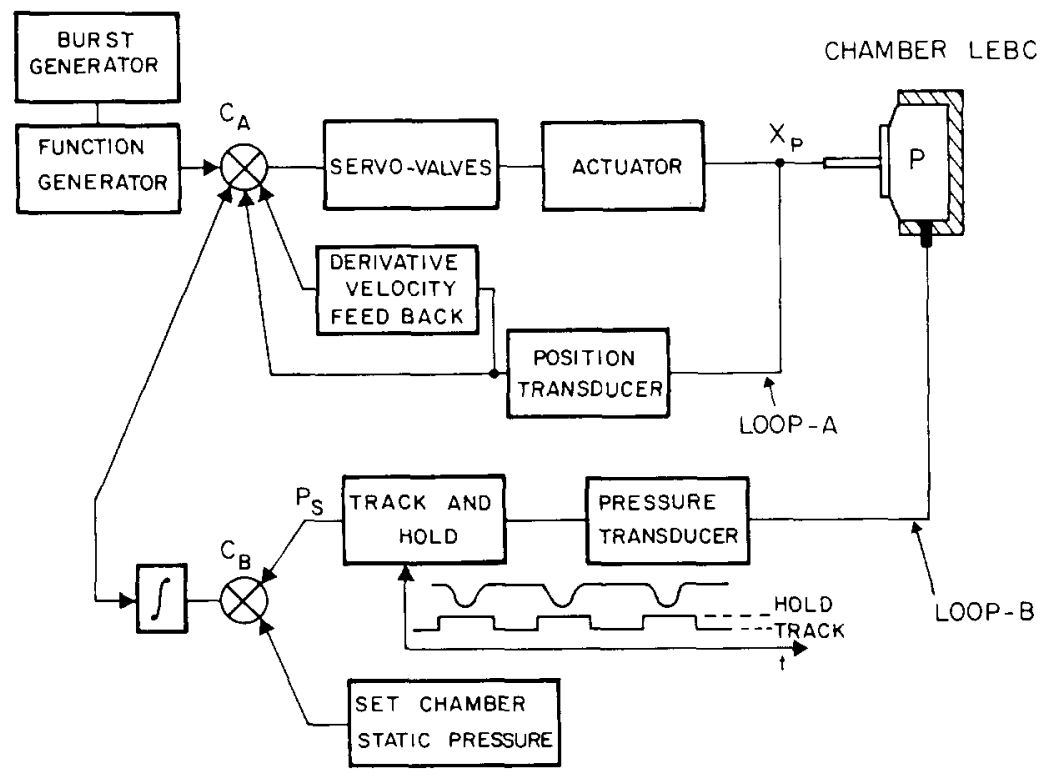

Fig. 6. Block diagram of the forced hydraulic expansion system. 


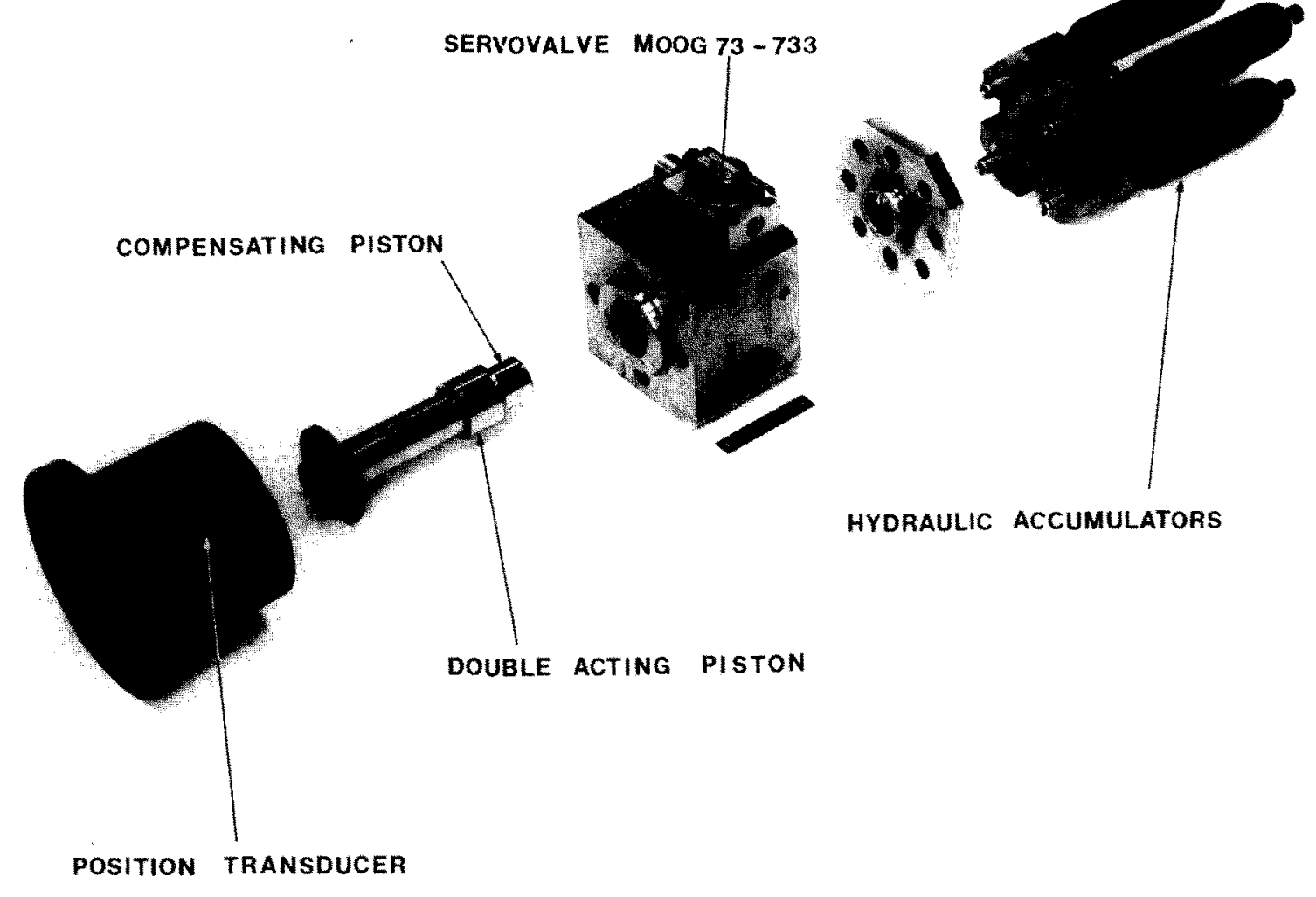

Fig. 7. Exploded view of the hydraulic expansion system.

\subsection{Refrigeration}

The basic design feature of the refrigeration system was dictated by the available construction time, which was limited to less than six months. Therefore, we cooled LEBC by forced circulation of two-phase hydrogen. This offered the advantages of isothermal refrigeration and simple temperature control, supplied from standard CERN dewars. To make this scheme feasible, careful attention had to be paid to

Table 3

Parameters for the hydraulic expansion actuator.
Moving mass (including cold piston)

Fastest complete expansion cycle

Maximum stroke from endstop to endstop

Maximum speed at zero fotce

Maximum force at zero speed
Maximum dynamic stroke

Nominal hydraulic pressure
$11 \mathrm{~kg}$

$5 \mathrm{~ms}$

$1.5 \mathrm{~mm}$

$3 \mathrm{~mm}$

$1.2 \mathrm{~ms}^{-1}$

$3520 \mathrm{daN}$

340 bar the total hydrogen consumption by minimising the conductive and radiant heat loads of the entire instal. lation.

A schematic diagram of the cooling circuit is shown in fig. 8 and the actual location of the equipment can be seen on the vertical cross section of the LEBC vacuum tank in fig. 4. Liquid hydrogen is continuously transferred from the pressurized storage dewar into the $20 \mathrm{l}$ container. This container serves as a phase separator for the saturated boiling liquid from the transfer-line and provides liquid hydrogen during the exchange of storage dewars. Then, the liquid flows through the heat exchanger which, inside the chamber volume, controls the hydrogen temperature and finally passes through a series of heat exchangers which intercept the radiant and conductive heat loads. During experiment NA13 we used a $180 \mathrm{\ell}$ hydrogen dewar with a $5 \mathrm{~m}$ transfer-line. To increase the autonomy during the longer exposures of experiment NA16, we replaced it by a $1000 \ell$ dewar. For safety reasons this was installed outside 


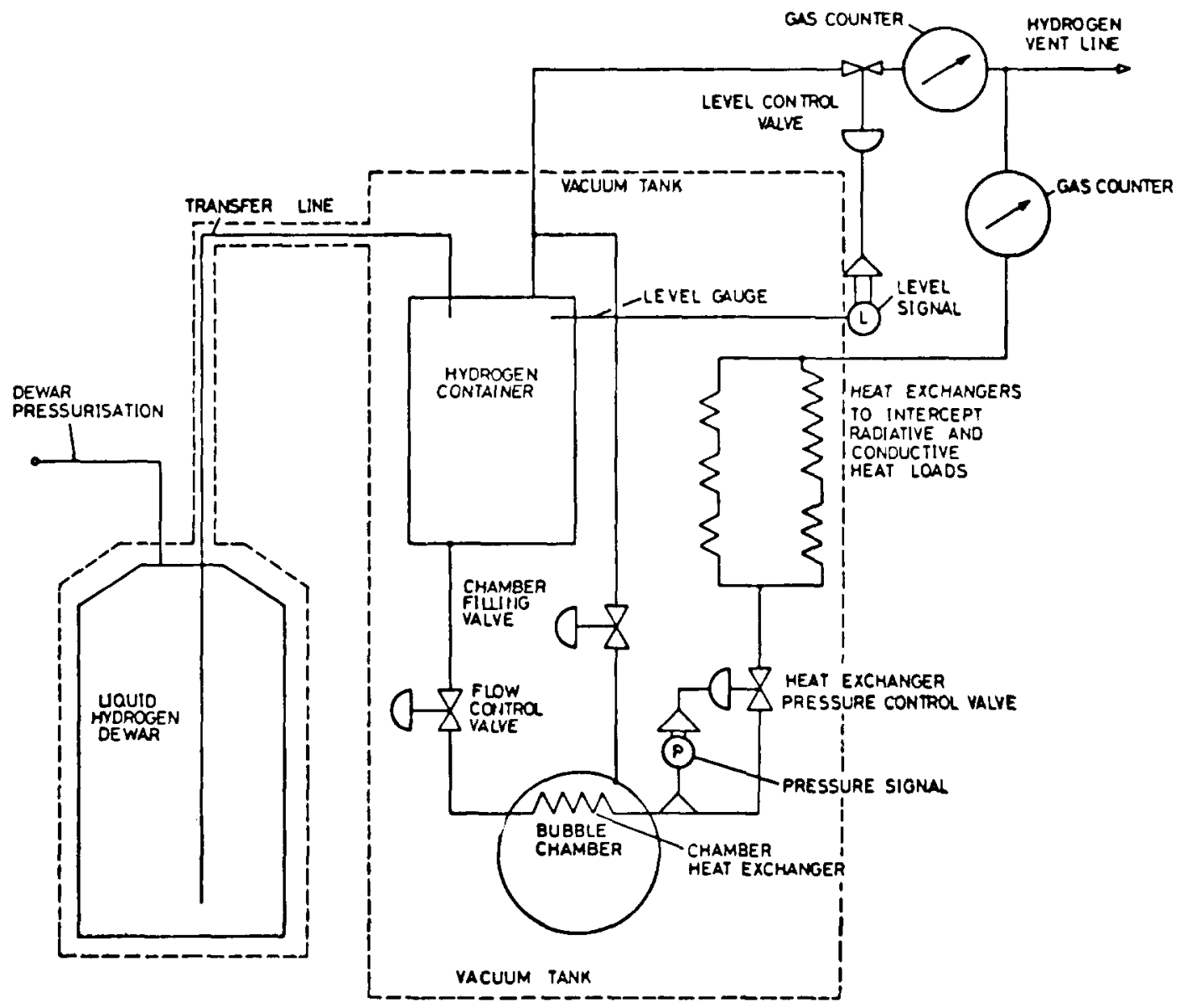

Fig. 8. Schematic diagram of cooling circuit.

the experimental hall and therefore required a longer liquid-nitrogen shielded transfer line $(20 \mathrm{~m})$.

The chamber internal heat exchanger (fig. 3 ) is essentially a $140 \mathrm{~cm}$ long stainless steel tube of $5 \mathrm{~mm}$ outer diameter and $0.5 \mathrm{~mm}$ wall thickness. Its inlet valve maintains an adequate mass flow and its outlet valve automatically controls, via the feed-back of a pressure pick-up, the temperature of the two-phase refrigerant. The hydrogen temperature in the chamber is then self-regulated at a $0.5 \mathrm{~K}$ higher level. To avoid any dirt inside the chamber it is filled by condensing cold gas from the container. This and the purging procedures require one single cold valve only, which considerably improves the reliability and simplicity of the system. During data taking, in its closed position, this cold valve had to seal the chamber volume perfectly, a sine qua non for a membrane expanded small chamber, which is extremely sensitive to the least loss of hydrogen. The absolute chamber temperature is determined from a vapour pressure bulb, but all temperature fluctuations are instantly and with high sensitivity indicated by corresponding changes of the piston rest-position: a piston movement of $0.1 \mathrm{~mm}$ corresponded to $0.02 \mathrm{~K}$ temperature variation.

The cool down of LEBC took around $24 \mathrm{~h}$, followed by $1 \mathrm{~h}$ filling time. Thermal stability was reached about $12 \mathrm{~h}$ after filling was completed. Satisfactory operating conditions at $29.1 \mathrm{~K}$ hydrogen temperature were achieved with the heat exhanger set at $28.6 \mathrm{~K}$ at a mass flow of $7 \times 10^{-2} \mathrm{gs}^{-1}$ provided by the dewar pressurized at 7.5 bar. The static heat load of the chamber was $5 \mathrm{~W}$. This value was obtained from the evaluated heat transfer rate due to the $0.5 \mathrm{~K}$ temperature difference between the liquid hydrogen in the chamber and in the heat exchanger. A dynamic 


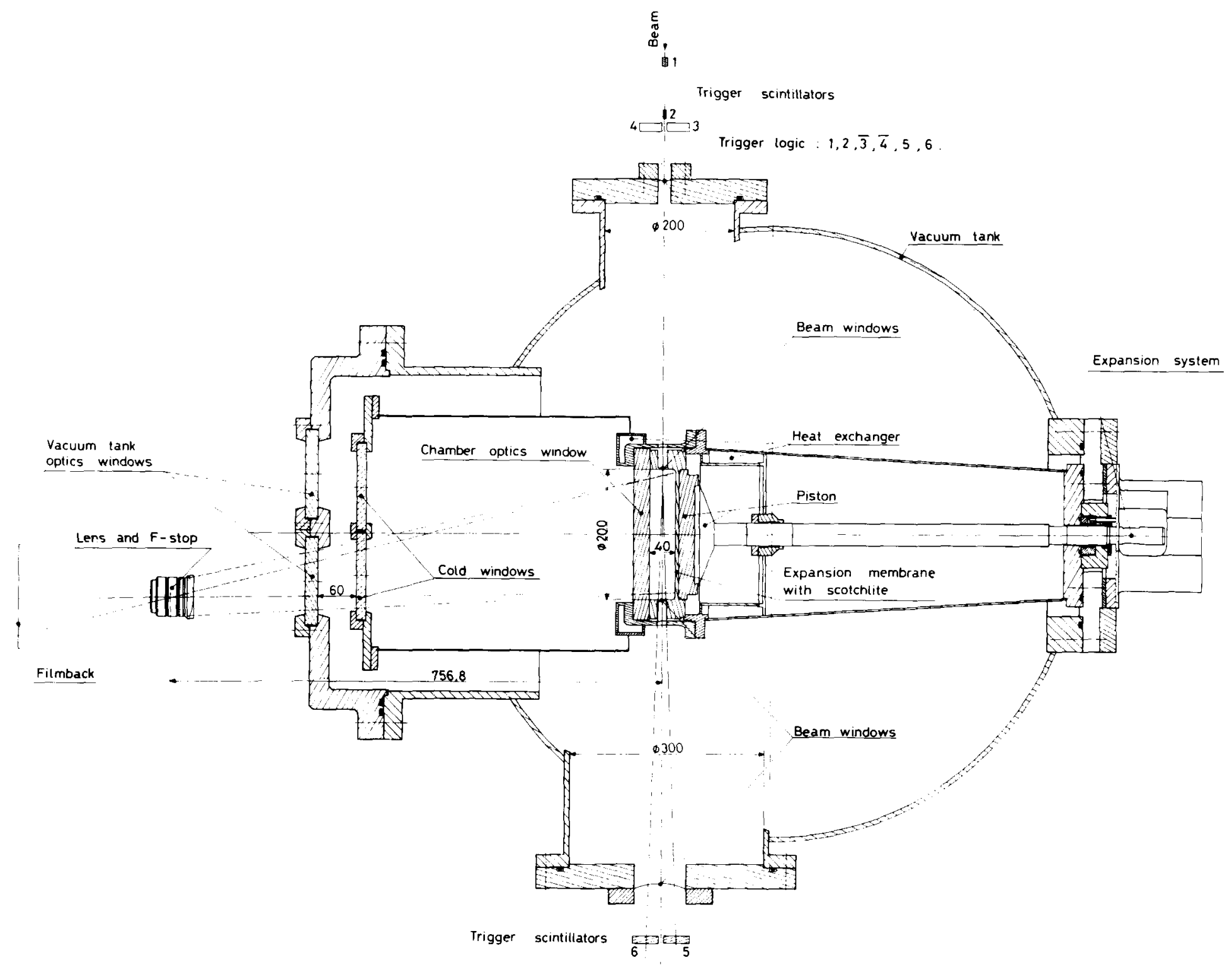

Fig. 9. Horizontal section of LEBC in its vacuum tank with optical arrangement and trigger logic. 
heat load of $0.1 \mathrm{~W}$ was derived from the small variation of the piston rest-position due to the increase of the hydrogen temperature after data taking had started, with the chamber cycling at $33 \mathrm{~s}^{-1}, 4 \mathrm{bar}$ pressure swings and $1.8 \mathrm{~s}$ cycling periods every $12 \mathrm{~s}$.

In spite of the unfavourable surface to volume of LEBC as compared with larger and therefore thermally more favourable chambers, we achieved a thermal stability of $\pm 0.01 \mathrm{~K}$ over more than $24 \mathrm{~h}$. However, after long periods of perfect stability we suddenly lost control of the temperature and the hydrogen in the chamber warmed up rapidly. We explain these unpleasant perturbations, which lasted for several minutes, by inadequate phase separation inside the hydrogen container, followed by an accumulation of gas, due to phase-separation inside the connecting pipes and a corresponding drop of massflow through the heat exchangers. Fortunately, these perturbations did not affect our data taking efficiency, but demanded increased attention from the shift team during these short periods.

\subsection{Optics and cameras}

The vertical and horizontal sections of the optics arrangement for LEBC are shown in figs. 4 and 9 , respectively. We employed retrodirective bright field photography with apertures set for high resolution and distances chosen for small demagnification. A point-like light source of $30 \mathrm{~J}$ electrical energy and 30 $\mu$ s flash duration at full width, half maximum, was installed between the vertically arranged pair of camera lenses (fig. 4). Its light was guided via two semi-transparent mirrors, mounted in a $45^{\circ}$ position in front of each lens, to simulate light originating from the two lens apertures. Then it was transmitted through the vacuum tank BK7-windows (at ambient temperature), the quartz cold windows (at about $100 \mathrm{~K}$ ), which served as radiation shields for the chamber, and finally through its Lexan-window into the hydrogen. From the Scotchlite screen, glued on the inner surface of the expansion membrane (fig. 3), it was retrodirected the same way back into the lenses, which were of the commercially available

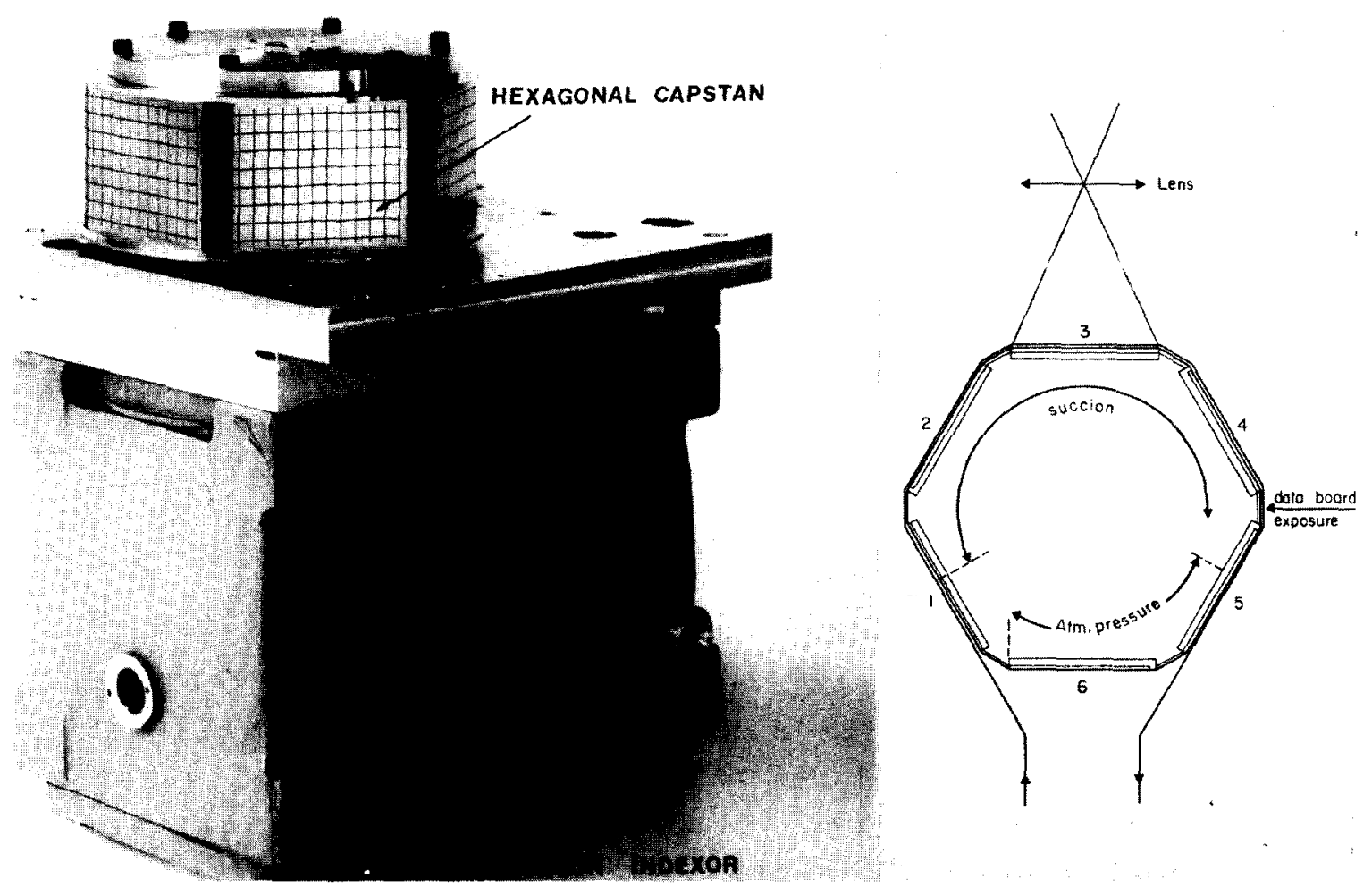

Fig. 10. Hexagonal capstan and Ferguson indexer for film transport and exposure. Film suck-down is provided in positions 2,3 (exposure) and 4 by a rotary valve, which also vents positions 5 and 6 to atmosphere in order to unstick the film. 
Componen-S * type. The focal lengths were $240 \mathrm{~mm}$ (NA13) and $180 \mathrm{~mm}$ (NA16) and the demagnifications 2.1 and 3.25 , respectively. With apertures $F / 16$ and $F / 11$ (NA16) the theoretical resolutions were about $40 \mu \mathrm{m}$ with $8 \mathrm{~mm}$ depth of field. Photographs were taken by triggering the flash-light with an electronic pulse, which was time delayed by $300 \mu$ s to allow for bubble growth. This pulse was released from the beam interaction trigger logic **, which is also shown in fig. 9.

The NA13-events were photographed with one camera only, which was a modified version of the CERN $2 \mathrm{~m}$ chamber and could be operated at a maximum rate of $8 \mathrm{~s}^{-1}$. The pictures for experiment NA16 were taken with modified cameras from the Rapid Cycling Bubble Chamber at CERN in two stereoscopic views. In each camera, the flash-light, retrodirected from the chamber, is focussed by the lens onto the $50 \mathrm{~mm}$ wide unperforated film *** mounted on an hexagonal capstan of $56 \mathrm{~mm}$ face lengths (fig. 10). To achieve the high speed film transport, required for the camera rate envisaged, a new drive mechanism was developed [9], which transports the film by rotating the hexagonal capstan stepwise through $60^{\circ}$, to bring its next face into position for photography. At the same time, a rotary valve distributes vacuum to the exposed (position 3 in fig. 10) and to the two adjacent faces (positions 2 and 4), to provide film suck-down, and vents the next faces (positions 5 and 6) to atmosphere in order to unstick the film. This design ensures complete film flatness during the exposure, since the same piece of film remains sucked down during three successive capstan positions. Good reproducibility of the capstan angular positions, after each $60^{\circ}$ rotation for film transport, is indispensable for producing well focussed pictures. It is achieved with the stout and precise Ferguson $\dagger$ indexing mechanism (fig. 10). The cameras were tested at a rate of $25 \mathrm{~s}^{-1}$, which corresponds to $40 \mathrm{~ms}$ dead time, and operated with $62 \mathrm{~ms}$ dead time in the trigger mode. The track residuals finally measured on the photographs indicate perfect film positioning.

Roll, trame and expansion number were printed on each frame by a data board, which was developed

\footnotetext{
* J. Schneider, Optische Werke, D-6550 Bad Kreuznach (Germany).

** Designed and built by Rome University (Italy).

***3 3921.

$\dagger$ Ferguson Machine Co., 33 Parc Industire, 1420 BraineLe-Château (Belgium).
}

in conjunction with the fast cameras. Light from a flash tube is encoded by relays $\dagger^{\dagger}$ and transmitted by optics fibers into a pseudo-contact printing head, which is placed at the rounded corner (fig. 10) of the hexagonal capstan $\dagger^{\dagger \dagger}$.

\section{Beam exposures and results}

LEBC construction was approved by the CERN Research Board on 16 November 1978 and six months later first tracks were photographed in the chamber. After one short test run, a production run, known as experiment NA13, was started in a 340 $\mathrm{GeV}$ negative pion beam and 110000 single view pictures were taken in June 1979. The interaction trigger (fig. 9) selected mainly those beam interactions in hydrogen, which occurred within $0.8 \mathrm{~ms}$ gate-time around the expansion cycle pressure minimum and initiated the flash-delay for bubble growth. Since the untimed beam particles were not removed by a kicker-magnet during the long spill extraction, a typical NA13 picture (fig. 11) has a number of early tracks with larger bubble diameters and lower bubble density, together with the triggered event with resolved bubble diameters from 45 to $55 \mu \mathrm{m}$ and 50 to $70 \mathrm{~cm}^{-1}$ bubble density. The physics results of experiment NA13 are published in ref. 6 .

Experiment NA13 was performed by exposing LEBC without any down-stream particle spectrometer. Therefore, in a second experiment (NA16), LEBC was placed at the vertex position of the European Hybrid Spectrometer (EHS), which provided momentum analysis, particle identification and gamma detection for secondaries emerging from the hydrogen interactions. For this exposure, LEBC was equipped with two fast cameras to permit stereoscopic event reconstruction and a kicker magnet was installed in the beam line, to remove all untimed beam particles. Therefore, in typical NA16 pictures (fig. 12) bubble sizes and bubble densities are rather uniform.

Several short test periods were required, to establish the alignment of all the spectrometer elements with the chamber fiducial volume in order to enable event reconstruction using the LEBC-pictures and the corresponding data acquired on magnetic tapes from the detection of gammas, $\mathrm{d} E / \mathrm{d} x$ measurements, wire

†† The driving mechanism was designed and built at Rome University (Italy).

$\dagger_{\dagger}$ More details of the data board are given in ref. [10]. 

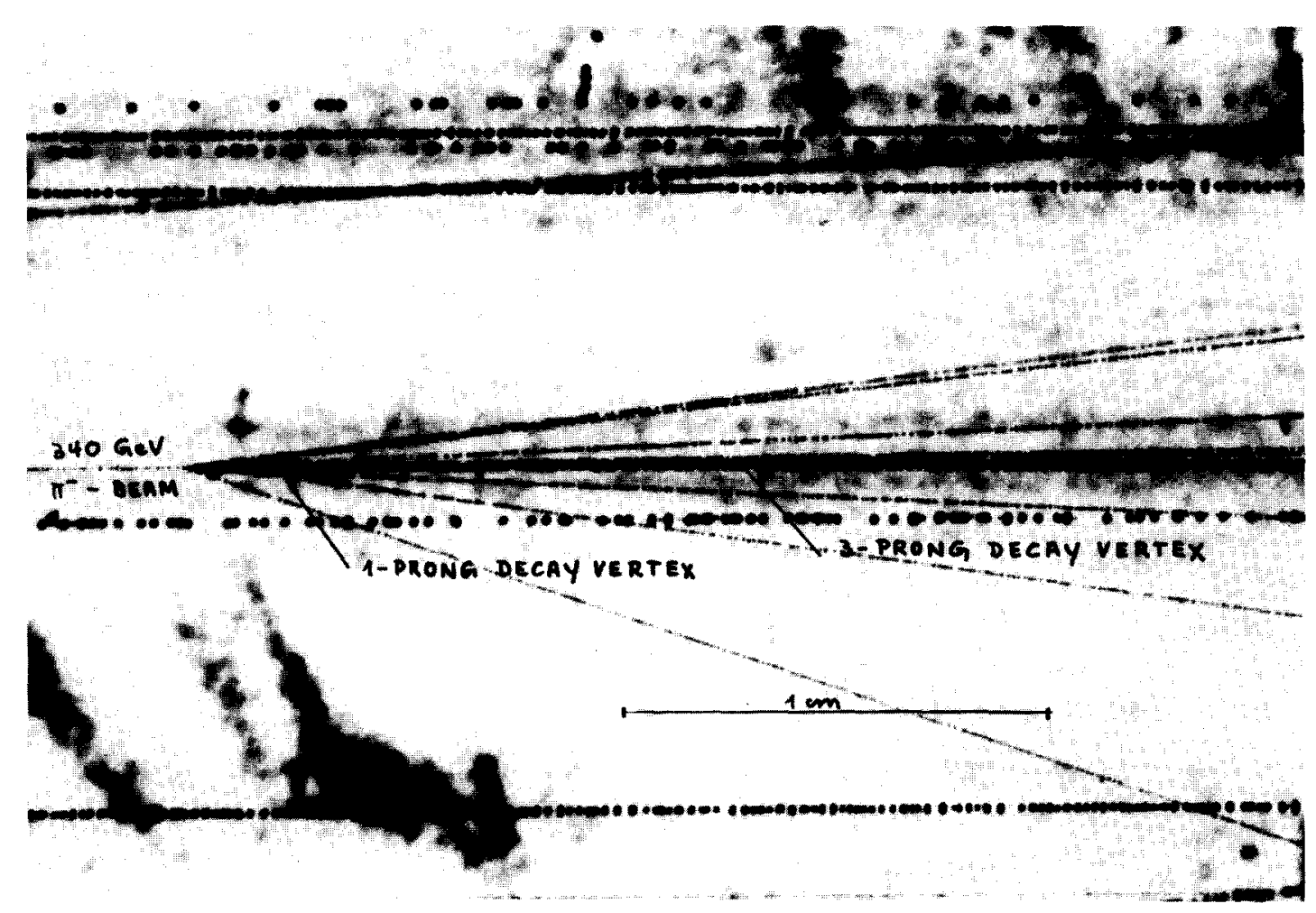

Fig. 11. NA13-picture with a $340 \mathrm{GeV} \pi^{-}$p interaction producing a pair of charged charm decays with $2.2 \mathrm{~mm}(1 \mathrm{prong})$ and 13.2 $\mathrm{mm}$ (3 prong) flight path. NA13-pictures were taken without kicker-magnet. Therefore, early track with big bubbles and low bubble density are visible.

and drift chamber coordinates. Production exposures began in April 1980 in a $360 \mathrm{GeV}$ negative pion beam, focussed to give an image of $80 \mathrm{~mm}$ height and $2 \mathrm{~mm}$ depth in the chamber, where 674000 pictures were taken. This was followed in June 1980 by a 360 $\mathrm{GeV}$ proton exposure, with the same beam image in the chamber, which resulted in 710000 pictures. Including all test runs, LEBC has taken a total of 1.63 million pictures in the two experiments and has been cycled for more than 20 million times. The availability of LEBC during the NA16 exposures was $92 \%$. Most of the dead time was required to change film rolls or hydrogen dewars and only about $1 \%$ of exposure time was lost on account of some minor thermal instabilities of the chamber. During the proton exposures we took an average number of 12.2 pictures per $1.8 \mathrm{~s}$ beam spill. The important operating parameters of LEBC for the NA1 6 exposure are listed in table 4.

Crucial parameters for the success of experiments NA13 and NA16 were resolved bubble sizes, bubble densities and track reconstruction accuracies. The diameters of bubbles on our LEBC photographs were firstly determined by direct measurements with a

microscope; from triggered events we found diameters between 37 and $45 \mu \mathrm{m}$ in real space. By a second method we scanned across the bubble images on film with a micro-densitometer. The measured full widths

Table 4

LEBC operating parameters for experiment NA16.

Hydrogen temperature

Hydrogen (equilibrium) vapor pressure

Hydrogen static pressure

Hydrogen pressure minimum

Hydrogen temperature fluctuations

Piston stroke

Length of expansion cycle

Chamber cycling rate

Camera cycling rate (max.)

SPS flat top

SPS cycle every

Beam gate at pressure minimum

Lens focal length

Demagnification

Aperture

Depth of field

Flash delay
$29.1 \mathrm{~K}$

$7.10 \mathrm{bar}$

8.15 bar

4.1 bar

$0.01 \mathrm{~K}$

$1.25 \mathrm{~mm}$

$5 \mathrm{~ms}$

$33 \mathrm{~s}^{-1}$

$17 \mathrm{~s}^{-1}$

$1.8 \mathrm{~s}$

$12 \mathrm{~s}$

$800 \mu \mathrm{s}$

$180 \mathrm{~mm}$

3.25

$\mathrm{F} / 11$

$5 \mathrm{~mm}$

$300 \mu \mathrm{s}$ 


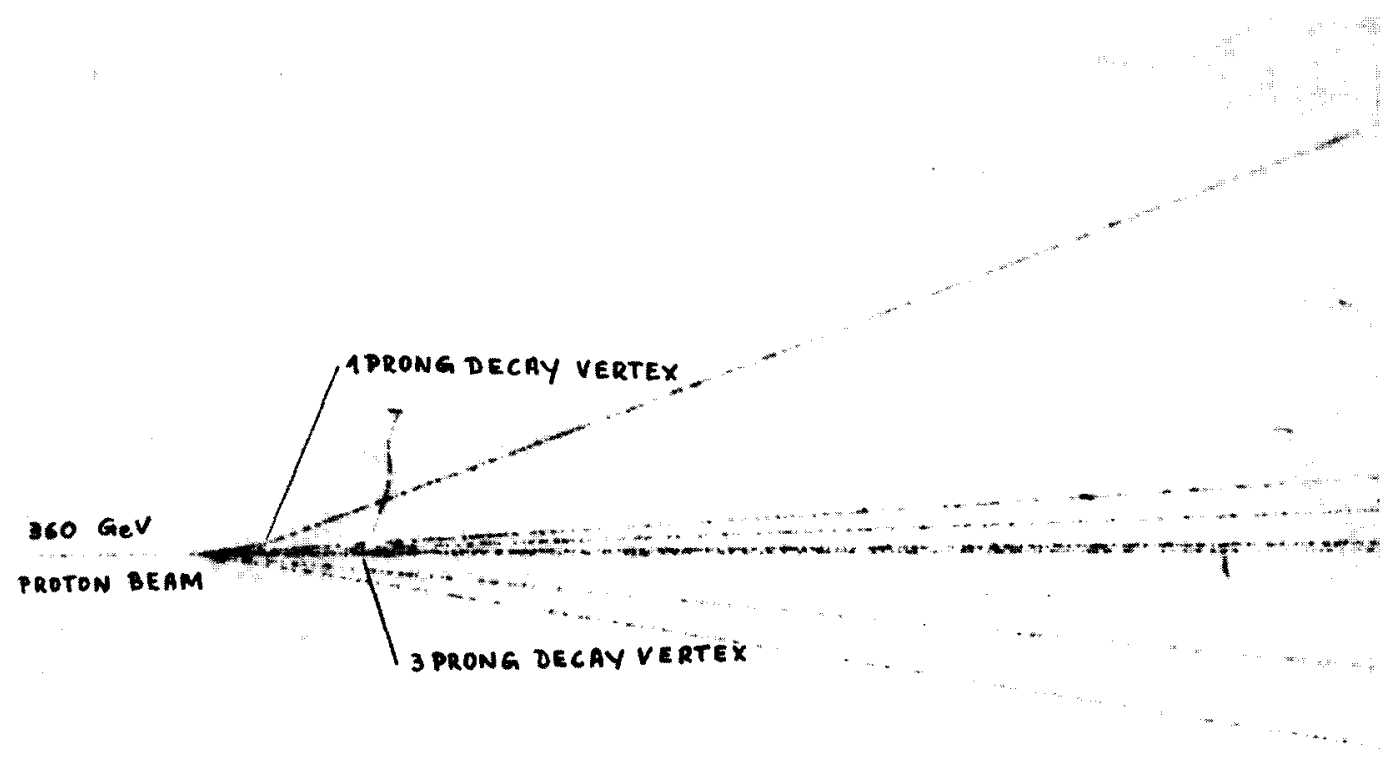

Fig. 12. NA16-picture with a $360 \mathrm{GeV}$ pp interaction producing a pair of charged charm decays with $0.7 \mathrm{~mm}(1 \mathrm{prong})$ and 2.4 $\mathrm{mm}$ (3 prong) decay length. The kicker-magnet has removed all particles not on time.

at half maximum of the more or less Gaussian shaped optical density curves yielded bubble diameters of $(42 \pm 6) \mu \mathrm{m}$ in space from triggered events. The rather large error margins associated with both methods indicate that our tracks are formed by bubbles of different sizes. This can be attributed to bubble coalescence during their growth-time. The diameter $\phi_{c}$ of two just-coalesced bubbles depends on the distance $d$ between their centres $\left(\phi_{\mathrm{c}}=2^{1 / 3} d\right)$ at formation. At the instant of picture taking, the different bubble diameters $\phi_{\mathrm{p}}$ span therefore the whole range between those for singly grown bubbles $\phi_{s}$ and those for $n$ bubbles coalesced just at that very moment $\left(\phi_{\mathrm{p}}=n^{1 / 3} \phi_{\mathrm{s}}\right)$. This means that blobs which result from coalescence of two bubbles, are photographed with diameters which depend on the original separation of these two bubbles and may exceed that of a single grown one by up to $26 \%$.

The probability of bubble coalescence can therefore be estimated from the mean bubble density. This quantity is also crucial for the observation efficiency of charm decays, since low bubble densities yield rather large gap lengths, which could make disentan- gling of multiprong vertices impossible and even simulate short range decays. To establish the bubble densities in our pictures, we counted about 15000 bubbles from triggered events having beam tracks more than $10 \mathrm{~cm}$ long in three different film rolls and found an average density of $(80.5 \pm 0.7) \mathrm{cm}^{-1}$. No evidence could be detected for a dependence of the bubble density either on the track location in the chamber or on the expansion number within the 67 cycles of a beam spill.

As is well known, the statistical fluctuations in bubble position along a particle track follow a Poisson distribution. For an original bubble density $b$ it describes the probability

$P(n)=(b x)^{n} \mathrm{e}^{-b x} / n !$

that $n$ bubbles are formed within a distance $x$. From relation (5) we calculated the probabilities $P(n)$ for different numbers of bubble $n=1,2,3, \ldots$, within $x=40 \mu \mathrm{m}$ distance, the average value of our resolved bubble diameters. The original bubble densities $b$ ranged from 70 to $120 \mathrm{~cm}^{-1}$. Based on these probabilities, we took into account bubble coalescence and 
Table 5

Relevant data of a $\mathrm{D}^{\circ} \overline{\mathrm{D}}^{\circ}$ pair, fully reconstructed in LEBC and EHS.

\begin{tabular}{lllll}
\hline Delay mode & $\begin{array}{l}\text { Mass } \\
\mathrm{MeV} / \mathrm{c}^{2}\end{array}$ & $\begin{array}{l}\text { Momentum } \\
\mathrm{GeV} / \mathrm{c}\end{array}$ & $\begin{array}{l}\text { Flight path } \\
\mathrm{mm}\end{array}$ & $\begin{array}{l}\text { light time } \\
10^{-13} \mathrm{~s}\end{array}$ \\
\hline $\mathrm{D}^{\circ} \rightarrow \mathrm{K}^{-} \pi^{+} \pi \pi^{\circ}$ & $1857 \pm 22$ & $11.90 \pm 0.6$ & $4.1 \pm 0.1$ & $2.1 \pm 0.1$ \\
$\mathrm{D}^{\circ} \rightarrow \mathrm{K}^{+} \pi^{+} \pi^{-} \pi^{-}$ & $1862 \pm 9$ & $78.5 \pm 0.3$ & $7.5 \pm 0.1$ & $5.9 \pm 0.1$ \\
\hline
\end{tabular}

evaluated the corresponding blob densities at the instant of picture taking. From the plot representing these two densities, we finally learned, that our counted density of $80.5 \mathrm{~cm}^{-1}$, which we now should rather call blob density, corresponds to $97 \mathrm{~cm}^{-1}$ original bubble density. The $80.5 \mathrm{~cm}^{-1}$ blob density is composed of $66 \mathrm{~cm}^{-1}$ single bubbles, $13 \mathrm{~cm}^{-1}$ blobs of two bubbles and $1.5 \mathrm{~cm}^{-1}$ blobs of three bubbles, i.e. $18 \%$ of all bubbles in our triggered events are blobs and should not be taken into account, when we determine the resolved bubble diameter.

The basic limit on the measurement accuracy of a particle track is its r.m.s. deviation from the expected trajectory, in our case, without magnetic field, from a straight line. This deviation represents a typical figure of merit for a bubble chamber, since it is affected by track displacements due to liquid motions, which are mainly induced by the expansion cycles. From 32000 measured beam tracks, $18 \mathrm{~cm}$ long each, we found $1.95 \mu \mathrm{m}$ r.m.s. residual on film which corresponds to $6.2 \mu \mathrm{m}$ in space. This value includes errors of the ERASME measuring machine at CERN, which are of the same magnitude. Larger chambers produced typical r.m.s. residuals of $50-70 \mu \mathrm{m}(2 \mathrm{~m}$ chamber) and 250-300 $\mu \mathrm{m}$ (BEBC) in space.

After the titles and the alignment of LEBC and the EHS-detectors have been established, particle trajectories starting from LEBC-vertices can be hybridized through the spectrometer once they stay within its geometrical acceptance. Meanwhile, data processing for the NA16-experiment is well advanced and several charm decays have been already detected and identified. As an example for the accuracies which can be achieved with the LEBC-EHS set-up, table 5 shows the relevant parameters of a $\left(D^{0}, \bar{D}^{0}\right)$ pair, which is published elsewhere [11]. A list of 21 fully reconstructed charmed decays was presented in Lisbon [12]. These events represent the initial results of scanning and measuring the first $300 \mathrm{k}$ pictures of NA16.
We are indebted to D. Güsewell, J. May, L. Mazzone, A. Minten, L. Montanet and H.P. Reinhard for their continuous interest and support during this work and we acknowledge the invaluable assistance of M. Dykes, Ch. Gregory, F. Pouyat, S. Reucroft, $R$. Settles and the trigger operating team under R. Bizzarri. To render a hydrogen bubble chamber operational within such a short period of time, the competence and the enthusiasm of the technical staff involved was crucial. Therefore, we would like to thank in particular H. Ancey, G. Dubail, A. Dupenloup, A. Francano, G. Geydet, R. Girardot, G. Merighi, G. Mouron, G. Pothier, A. Theron, M. Thevenon, L. Veillet and G. Waurick for their skilful and dedicated work during the construction and running of LEBC.

\section{References}

[1] G. Goldhaber et al., Phys. Rev. Lett. 37 (1976) 255; I; Peruzzi et al., Phys. Rev. Lett. 37 (1976) 569.

[2] DASP Collaboration, Phys. Lett. 63B (1976) 471; 70B (1977) 125 .

[3] L. Voyvodic, Invited talk Intern. Symp. on Lepton and photon interactions, FNAL, 1979; M. Conversi, Review talk, EPS Conf. on High Energy Physics, Geneva, 1979.

[4] Photon Emulsion $+\Omega$-Photon Collaboration: Phys. Lett. 89B (1980) 427.

[5] J.L. Ritchie et al., Phys. Rev. Lett. 44 (1980) 230; P. Alibran et al,, Phys. Lett. 74B (1978) 134; T. Hansl et al., Phys. Lett. 74B (1978) 139; P. Bosetti et al., Phys. Lett. 74B (1978) 143.

[6] W. Allison et al., Phys. Lett. 93B (1980) 509.

[7] D. Crennel et al., Nucl. Instr. and Meth. 158 (1979) 111.

[8] R.M. Waxler, D. Horovitz and A. Feldmann, Appl. Optics 18 (1979) 101.

[9] J.L. Benichou, A. Hervé and G. Merighi, Internal report CERN/EF/EHS/TE 78-5 (1978).

[10] J.L. Benichou, D. French, A. Hervé and G. Merighi, Internal report CERN/EF/EHS/TE 78-6 (1978).

[11] LEBC-EHS Collaboration, Phys. Lett. B (1981) in press.

[12] LEBC-EHS Collaboration, Proc. Int. Conf. on High Energy Physics, Lisbon 1981. 